\title{
ON QUANTUM WEYL ALGEBRAS AND GENERALIZED QUONS
}

\author{
WŁADYSEAW MARCINEK \\ Institute of Theoretical Physics, University of Wroctaw \\ Pl. Maksa Borna 9, 50-204 Wroctaw, Poland \\ E-mail:wmar@ift.uni.wroc.pl
}

\begin{abstract}
The model of generalized quons is described in an algebraic way as certain quasiparticle states with statistics determined by a commutation factor on an abelian group. Quantization is described in terms of quantum Weyl algebras. The corresponding commutation relations and scalar product are also given.
\end{abstract}

1. Introduction. It is well known that all standard particles in physics like electrons, photons, protons and all others can be divided into two classes according to their statistics: fermions and bosons. In the last years particle excitations equipped with new kind of statistics have been discovered. For example particles with fractional spin and statistics interpolating between fermions and bosons appears in the two dimensional field theories $[1,2,3,4,5,6]$. In this paper we are going to study a generalized quons as a generalization of Wilczek model of anyons. For our generalized quons we obtain the following relations

$$
\begin{array}{ll}
a_{i}^{*} a_{i}-q_{i} a_{i} a_{i}^{*}=1, & a_{i}^{*} a_{j}-b_{i j} a_{j} a_{i}^{*}=0, \text { for } i \neq j, \\
a_{i} a_{j}-b_{i j} a_{j} a_{i}=0, & a_{i}^{*} a_{j}^{*}-b_{i j} a_{j}^{*} a_{i}^{*}=0,
\end{array}
$$

where $-1 \leq q_{i} \leq 1$ are real parameters, and $\bar{b}_{i j}=b_{j i}$ are some coefficients.

2. Generalized quons. Let us consider a particle moving on a plane with $N$ singular points. The proper physical nature of such singularities is not important for us. Only discrete rotations of this particle around singularities are essential for our model. The moving between singularities is completely irrelevant. Hence it is not difficult to show that the rotational excitations on singularities behave like a system of $N$ independent particles. These rotational excitations are called generalized quons. In this Section we are

1991 Mathematics Subject Classification: 81R50, 81S05, $17 \mathrm{~B} 37$.

The paper is partially supported by KBN, Grant No 2P 30208706.

The paper is in final form and no version of it will be published elsewhere. 
going to describe the Weyl algebra for our system of generalized quons. We assume that the $i$-th singular point is characterized by a real numbers $q_{i},-1 \leq q_{i} \leq 1, i=1, \ldots, N$. We also assume that $\sigma_{i}$ is an operator for discrete rotation around the $i$-th singular point. Next we assume for simplicity that $\sigma_{i},(i=1, \ldots, N)$ generate an abelian group $\Gamma$. For rotations on $2 \pi$ angle we have $\Gamma=Z \oplus, \ldots, \oplus Z,(N$-sumands) for example. It is natural to assume that the statistics for our quons is determined by a commutation factor in the sense of Scheunert [7] on the group $\Gamma$. Recall that a commutation factor on an abelian group $\Gamma$ is a mapping $b: \Gamma \times \Gamma \longrightarrow \mathbf{C}-\{0\}$ such that

$$
b(\alpha+\beta, \gamma)=b(\alpha, \gamma) b(\beta, \gamma), \quad b(\alpha, \beta) b(\beta, \alpha)=1
$$

for every $\alpha, \beta, \gamma \in \Gamma$. We assume here in addition that $b(\alpha, \alpha)=1$ for every $\alpha \in \Gamma$.

Let us give a short introduction to the concept of quantum Weyl algebras $[8,9]$ for the description of quantum states of our generalized quons. Let $E$ be a finite dimensional Hilbert space equipped with a basis $x^{i}, i=1, \ldots, N=\operatorname{dim} H$. The complex conjugate space is denoted by $E^{*}$. Note that our notation is not covariant. We assume that the pairing (.|.) : $E^{*} \otimes E \longrightarrow \mathbf{C}$ and the corresponding scalar product is given by

$$
\left(u^{*} \mid v\right) \equiv<u \mid v>:=\Sigma_{i=1}^{N} \bar{u}^{i} v^{i},
$$

where $u^{*}=\sum_{i=1}^{N} \bar{u}^{i} x^{* i}$ and $v=\sum_{i=1}^{N} v^{i} x^{i}$. We assume that the element $x^{i}$ describe the quantum state corresponding to one left rotation around the $i$-th singular point, i.e $x^{i}$ corresponds to the generator $\sigma_{i}$. In other words the space $E$ is graded by $\Gamma$, i.e. $x^{i}$ is a homogeneous element with respect to the gradation of grade $\sigma_{i}$. Similarly $x^{* i}$ correspond to the one right rotation around the $i$-th singularity. For the gradation we have that $\operatorname{grade}\left(x^{i}\right)+\operatorname{grade}\left(x^{* i}\right)=0$. In this way the Hilbert space $E$ can be understood as the space of quantum states for left rotations and $E^{*}$ - for the right rotations. We have here the following

Definition 2.1. An algebra generated by $x^{i}$ and $x^{* j} ;(i, j=1, \ldots, N)$ subject to relations

$$
\begin{aligned}
& x^{* i} x^{i}=1+c_{i i} x^{i} x^{* i} \quad \text { for } i=j, \quad x^{* i} x^{j}=c_{j i} x^{j} x^{* i} \quad \text { for } i \neq j, \\
& x^{i} x^{j}=b_{i j} x^{j} x^{i} \quad \text { for all } i, j, x^{* j} x^{* i}=\bar{b}_{j i} x^{* i} x^{* j} \quad \text { for all } i, j \text {, }
\end{aligned}
$$

(no sum), for $i, j=1, \ldots, N$, where $c_{i j}$ are complex parameters such that $c_{i i} \equiv q_{i}, q_{i}$ characterize the $i$-th singular point on a plane, $c_{i j}=b_{i j}$ for $i \neq j$ and $b_{i j}:=b\left(\sigma_{i}, \sigma_{j}\right)$ are coefficient for the commutation factor $b$ on the grading group $\Gamma$, is said to be a quantum Weyl algebra $\mathcal{W}_{q, b}(N)$ for quons.

We introduce two operators $C$ and $B$ by relations

$$
C\left(x^{* i} \otimes x^{j}\right):=c_{j i} x^{j} \otimes x^{* i}, \quad B\left(x^{i} \otimes x^{j}\right):=b_{i j} x^{j} \otimes x^{i}, \quad \text { (no sum) }
$$

Let us introduce a linear operator $R_{n}$ by the formula

$$
R_{n}:=i d+\tilde{C}^{(1)}+\tilde{C}^{(1)} \tilde{C}^{(2)}+\ldots+\tilde{C}^{(1)} \ldots \tilde{C}^{(n-1)},
$$

where $\tilde{C}^{(i)}:=i d_{E} \otimes \ldots \otimes \tilde{C} \otimes \ldots \otimes i d_{E}, \tilde{C}$ on the i-th place, and $(\tilde{C})_{k l}^{i j}=C_{l * j}^{* k i}$. Let us consider a few simple examples: 
EXAMPLE 2.1. In the case of $N=1$ we obtain

$$
x^{*} x=q x x^{*}+1,
$$

i.e. the usual $q$-commutation relations, see Ref. [4] for example. The corresponding quantum Weyl algebra is denoted by $\mathcal{W}_{q}$. Here we have $\Gamma=Z$, and $\operatorname{grade}(x)=-$ $\operatorname{grade}\left(x^{*}\right)=1$.

EXAMPLE 2.2. For $N=2$ we have an algebra generated by $x, y$ and $x^{*}, y^{*}$ and relations

$$
x^{*} x=q x x^{*}+1, y^{*} y=p y y^{*}+1, x y= \pm y x,
$$

where $q_{1}=q, q_{2}=p$, and $b_{i j}= \pm 1, \Gamma=Z \oplus Z$.

ExAmple 2.3. For arbitrary $N \geq 2$ we can define an algebra $\mathcal{W}_{q, \Theta}(N)$ for which we have

$$
b_{i j}=\exp \frac{2 \pi \Theta_{i j}}{n}
$$

where $\Theta_{i j}=-\Theta_{j i}, \Theta_{i j}$ is an integer $(\bmod \mathrm{n})$ for every $i, j=1, \ldots, N, i \neq j$, and $q=\left\{q_{i} \mid-1 \leq q_{i} \leq 1, i=1, \ldots, N\right\} \Gamma=Z_{n} \oplus, \ldots, \oplus Z_{n}$. The case $q_{i}=1$ for every $i=1, \ldots, N$ corresponds to particles which are in usual called anyons.

3. The category of states and Fock representation. In this Section we would like to describe all quantum states for the system of generalized quons. As was indicated in the previous Section, spaces $E$ and $E^{*}$ correspond for the left and right single rotations around singularities. For multiply rotations we need tensor product of these spaces. In this way all states can be described by a family of spaces. Such family forms a braided monoidal category. In fact if we have two linear and invertible operators $C: E^{*} \otimes E \longrightarrow$ $E \otimes E^{*}$, and $B: E \otimes E \longrightarrow E \otimes E$, then under some technical assumtions there is a braided monoidal category $\mathcal{C} \equiv(\otimes, k, \Psi)$ generated by $E, E^{*}, C$ and $B,[10,11,12]$. The category $\mathcal{C}$ contains: the underground field $k=\mathbf{C}$ of complex numbers, the vector space $E$ and the dual $E^{*}$, all tensor products of $E$ and $E^{*}$, all direct sums, and some quotients. We define braidings $\Psi_{E, E}, \Psi_{E^{*}, E}, \Psi_{E, E^{*}}$, and $\Psi_{E^{*}, E^{*}}$ by formulae

$$
\Psi_{E, E} \equiv B, \Psi_{E^{*}, E} \equiv C, \Psi_{E, E^{*}} \equiv C^{-1}, \Psi_{E^{*}, E^{*}} \equiv B^{*},
$$

where $\left(B^{*}\right)_{k l}^{i j}=B_{* j * i}^{* l * k}$. All above braidings can be extended to all tensor products of spaces $E$ and $E^{*}$. In this way we obtain

$$
\begin{aligned}
& \Psi_{E, E^{\otimes l}}:=B^{(l)} \circ \ldots \circ B^{(1)}, \\
& \Psi_{E^{\otimes k}, E^{\otimes l}}:=\Psi_{E, E^{\otimes l}}^{(1)} \ldots \circ \Psi_{E, E^{\otimes l}}^{(k)}, \\
& \Psi_{E^{* \otimes k}, E^{\otimes l}}, \\
& :=C^{(l)} \circ \ldots \circ C^{(1)} \circ \ldots \circ C^{(k+l-1)} \circ \ldots \circ C^{(k-1)} \circ C^{(k+l)} \circ \ldots \circ C^{(k)} .
\end{aligned}
$$

If we substitute the formula (4) into relations (9), then we obtain the braid symmetry and the corresponding braided monoidal category for our quons system. Note that if we have $q_{i}=1$ for every $i=1, \ldots, N$, then the category becomes symmetric! We can understand objects of the category as all possible spaces of quantum states for our quons. For example the tensor product $E^{\otimes k}$ can be understand as a space for all quantum states corresponding to the $k$ rotations to the left. 
An algebra $\mathcal{A} \equiv \mathcal{A}_{B}(E):=T E / I_{B}$, is said to be $B$-symmetric algebra over $E$ [16], if it is generated by the relation

$$
x^{i} x^{j}=b_{i j} x^{j} x^{i}, \quad \text { (no sum). }
$$

This algebra can be regarded as the algebra of all braid equivalent quantum states for left rotations. On the other hand we have a $B$-symmetric algebra $\mathcal{A}^{*}$ over the space $E^{*}$, it is an algebra defined as the quotient $\mathcal{A}^{*} \equiv \mathcal{A}_{B}\left(E^{*}\right):=T E^{*} / I^{*}$, such we have the following relations

$$
x^{* i} x^{* j}=b_{i j} x^{* j} x^{* i} .
$$

Obviously $\mathcal{A}$ and $\mathcal{A}^{*}$ are graded, quadratic, associative and unital algebras over $E$, and $E^{*}$, respectively, see Baez [16]. We denote by $P: T E \longrightarrow \mathcal{A}$ and $P^{*}: T E^{*} \longrightarrow \mathcal{A}^{*}$ the coresponding quotient mappings.

Definition 3.1. We define a right contraction as a family of linear mappings $c_{k}$ : $E^{*} \otimes \mathcal{A}^{k} \longrightarrow \mathcal{A}^{k-1}$ by the following formulae

$$
\begin{aligned}
& c_{1}\left(x^{* i} \otimes x^{j}\right):=\left(x^{* i} \mid x^{j}\right)=\delta^{i j}, \\
& c_{k}\left(x^{* i} \otimes x^{j} f\right):=c_{1}\left(x^{* i} \otimes x^{j}\right) f+c_{i j} x^{j} c_{k-1}\left(x^{* i} \otimes f\right),
\end{aligned}
$$

for $f \in \mathcal{A}^{k-1}$.

One can see that the above definition is consistent, see [19]. The left contraction mapping can be also defined. Note that in the above contraction is the same as the evaluation mapping from the previous papers, see Ref.[17, 18]. One can see that the contraction $c$ is well defined on the algebra $\mathcal{A}$. Observe that for the contraction $c$ we also have

$$
c_{k-1}\left(x^{* i} \otimes c_{k}\left(x^{* j} \otimes f\right)\right)-b_{i j} c_{k-1}\left(x^{* j} \otimes\left(c_{k}\left(x^{* i} \otimes f\right)\right)=0 .\right.
$$

on $E^{*} \otimes E^{*} \otimes \mathcal{A}^{k}$. We are going to construct a representation $a: \mathcal{W}_{q, b}(N) \longrightarrow \operatorname{End}(\mathcal{A})$ of the quantum Weyl algebra $\mathcal{W}$ on the algebra $\mathcal{A}$. For generators $x^{i}$ and $x^{* j},(i, j=1, \ldots, N)$ we define

$$
a_{x^{i}} f:=m\left(x^{i} \otimes f\right) \equiv x^{i} f, a_{x^{* i}} f \equiv c\left(x^{* i} \otimes f\right)
$$

for every $f \in \mathcal{A}$. One can extend this representation for the whole algebra $\mathcal{W}$. One can also consider the representation of the quantum Weyl algebra $\mathcal{W}$ on the algebra $\mathcal{A}^{*}$ in a similar way. We have here the following important

TheOREM 3.1. We have on the algebra $\mathcal{A}$ the following commutation relations for the representation of Weyl algebra $\mathcal{W}_{q, b}(N)$

$$
\begin{aligned}
& a_{x^{* i}} a_{x^{j}}-c_{i j} a_{x^{j}} a_{x^{* i}}=\delta^{i j}, \\
& a_{x^{i}} a_{x^{j}}-b_{i j} a_{x^{j}} a_{x^{i}}=0, a_{x^{* i}} a_{x^{* j}}-b_{i j} a_{x^{* j}} a_{x^{* i}}=0,
\end{aligned}
$$

Pro of. Using the relations (14) for the first relation (15) we obtain

$$
\begin{aligned}
& {\left[a_{x^{*} i} a_{x^{j}}-c_{i j} a_{x^{*} j} a_{x^{i}}\right] f} \\
& =\left[c_{l+1}\left(x^{* i} \otimes x^{j} f\right)-c_{i j} x^{j} c_{l}\left(x^{* i} \otimes f\right)\right]=c_{1}\left(x^{* i} \otimes x^{j}\right) f=\delta^{i j} f,
\end{aligned}
$$

where $f \in \mathcal{A}^{l}$ and the relation (12) has been used.

We introduce here the following state vector

$$
\left|n_{1} \ldots n_{N}\right\rangle=\left(x^{1}\right)^{n_{1}} \ldots\left(x^{N}\right)^{n_{N}}
$$


up to some normalization factor. For the ground state vector $\mid 0>\equiv \mathbf{1}_{\mathcal{A}}$ we have as usual $a_{x^{*} i} \mid 0>=0$. The scalar product can be given by the following formula

$$
<s\left|t>_{C}:=\delta_{m n}<s\right| P_{n} t>
$$

for $s \in \mathcal{A}^{m}$ and $t \in \mathcal{A}^{n}$, where $P_{n}$ is an operator defined by induction

$$
P_{n+1}:=\left(i d \otimes P_{n}\right) \circ R_{n},
$$

where $P_{1} \equiv i d$ and the operator $R_{n}$ is given by the formula (5). Note that there is a very interesting result of Bożejko and Speicher [20]. If the operator $\tilde{C}$ is a bounded operator acting on some Hilbert space such that

$$
\tilde{C}=\tilde{C}^{*},\|\tilde{C}\| \leq 1, \tilde{C} \text { is Yang-Baxter, }
$$

then the scalar product given by the relation (18) is positive definite. It follows from the mentioned above theorem of Bożejko and Speicher that the corresponding scalar product for our generalized guons is positive definite if all $q_{i}$ are real, $-1 \leq q_{i} \leq 1$, and $\bar{b}_{i j}=b_{i j}$. Note that in our quonic interpretation the vector $\mid n_{1} \ldots n_{N}>$ describe the state corresponding to $n_{1}$ left rotations corresponding to the first singularity,..., and to $n_{N}$ left rotations corresponding to the last singularity. It is interesting that creation and anihilation operators create and anihilate rotations (or more precisely - rotational excitations) not particles! Observe that in the above representation we have only left rotations. For right rotations we need the representation on the algebra $\mathcal{A}^{*}$.

\section{References}

[1] F. Wilczek, Phys. Rev. Lett. 48, 114 (1982).

[2] O. W. Greenberg, Phys. Rev. Lett. 64705 (1990), Phys. Rev. D 43, 4111 (1991).

[3] Y. S. Wu, Phys. Rev. Lett. 52, 2103 (1984), Phys. Rev. Lett. 53, 111 (1984).

[4] D. I. Fivel, Phys. Rev. Lett. 65, 3361, (1990).

[5] D. Zagier, Commun. Math. Phys. 147, 199 (1992).

[6] M. Bożejko, R. Speicher, Interpolation between bosonic and fermionic relations given by generalized Brownian motions. Preprint FSB 132-691, Heildelberg (1992)

[7] M. Scheunert, J. Math. Phys. 20, 712, (1979).

[8] E. E. Demidov, On some aspects of the theory of quantum groups, Uspekhi Mat. Nauk 48, 39, (1993), in Russ.

[9] A. Borowiec, V. K. Kharchenko, Z. Oziewicz, Calculi on Clifford-Weyl and exterior algebras for Hecke braiding, Conferencia dictata por Zbigniew Oziewicz at Centro de Investigation en Matematicas, Guanajuato, Mexico, Seminario de Geometria, jueves 22 de abril de 1993 and at the Conference on Differential Geometric Methods, Ixtapa, Mexico, September 1993.

[10] S. Majid, J. Geom. Phys. 13, 169, (1994).

[11] W. Marcinek, On unital braidings and quantization, Preprint ITP UWr No 847 (1993) and Rep. Math. Phys. 34, 325, (1994).

[12] W. Marcinek, Noncommutative geometry corresponding to arbitrary braidings, J. Math. Phys. 35, 2633, (1994).

[13] J. C. Baez, R-commutative geometry and quantization of Poisson algebras, Adv. Math. 95, 61 (1992). 
[14] R. Rałowski, On deformations of commutation relation algebras, Preprint IFT UWr $\mathbf{8 9 0}$, (1995), q-alg/9506004.

[15] W. Marcinek, On the deformation of commutation relations, in Proceedings of the XIII Workshop in Geometric Methods in Physics, July 1-7, 1994 Białowieża, Poland, ed. by J-P. Antoine et al, Plenum Press 1995.

[16] J. C. Baez, Lett. Math. Phys. 23, 1333, (1991).

[17] W. Marcinek and R. Rałowski, Particle operators from braided geometry, in "Quantum Groups, Formalism and Applications" XXX Karpacz Winter School in Theoretical Physics, 1994, Eds. J. Lukierski et al., 149-154 (1995).

[18] W. Marcinek and Robert Rałowski, On Wick Algebras with Braid Relations, Preprint IFT UWr 876/9, (1994) and J. Math. Phys. 36, 2803, (1995).

[19] W. Marcinek, On Commutation Relations for Quons, q-alg/9512015.

[20] M. Bożejko, R. Speicher, Math. Ann. 300, 97, (1994). 